\title{
Membrane topology analysis of HIV-1 envelope glycoprotein gp41
}

Shujun Liư ${ }^{1 \dagger}$, Naoyuki Kondo ${ }^{1,2,3+}$, Yufei Long ${ }^{1}$, Dan Xiao ${ }^{1}$, Aikichi Iwamoto ${ }^{3}$, Zene Matsuda ${ }^{1,2^{*}}$

\begin{abstract}
Background: The gp41 subunit of the HIV-1 envelope glycoprotein (Env) has been widely regarded as a type I transmembrane protein with a single membrane-spanning domain (MSD). An alternative topology model suggested multiple MSDs. The major discrepancy between the two models is that the cytoplasmic Kennedy sequence in the single MSD model is assigned as the extracellular loop accessible to neutralizing antibodies in the other model. We examined the membrane topology of the gp41 subunit in both prokaryotic and mammalian systems. We attached topological markers to the C-termini of serially truncated gp41. In the prokaryotic system, we utilized a green fluorescent protein (GFP) that is only active in the cytoplasm. The tag protein (HaloTag) and a membrane-impermeable ligand specific to HaloTag was used in the mammalian system.
\end{abstract}

Results: In the absence of membrane fusion, both the prokaryotic and mammalian systems (293FT cells) supported the single MSD model. In the presence of membrane fusion in mammalian cells (293CD4 cells), the data obtained seem to support the multiple MSD model. However, the region predicted to be a potential MSD is the highly hydrophilic Kennedy sequence and is least likely to become a MSD based on several algorithms. Further analysis revealed the induction of membrane permeability during membrane fusion, allowing the membrane-impermeable ligand and antibodies to cross the membrane. Therefore, we cannot completely rule out the possible artifacts. Addition of membrane fusion inhibitors or alterations of the MSD sequence decreased the induction of membrane permeability.

Conclusions: It is likely that a single MSD model for HIV-1 gp41 holds true even in the presence of membrane fusion. The degree of the augmentation of membrane permeability we observed was dependent on the membrane fusion and sequence of the MSD.

\section{Background}

The envelope glycoprotein (Env) of human immunodeficiency virus type-1 (HIV-1) plays a critical role in the early stage of HIV-1 infection. Env is synthesized as a precursor protein, gp160 [1,2], and processed into gp120 and gp41 during transport from the endoplasmic reticulum to Golgi network [3,4]. The gp120 subunit determines host range through its recognition of the receptor and co-receptor complex. The transmembrane protein gp41 mediates the membrane fusion between the host and viral membranes. It is composed of an ectodomain (extracellular domain), a cytoplasmic domain, and a

\footnotetext{
* Correspondence: zmatsuda@ims.u-tokyo.ac.jp

+ Contributed equally

'China-Japan Joint Laboratory of Structural Virology and Immunology, Institute of Biophysics, Chinese Academy of Sciences, 15 Datun Road,

Chaoyang District, Beijing 100101, P. R. China

Full list of author information is available at the end of the article
}

transmembrane domain. The ectodomain has coiledcoil-forming heptad repeats essential for membrane fusion. The cytoplasmic domain contains three amphipathic helices called the lentiviral lytic peptide (LLP) 1 , 2 and 3. The LLP-1 and LLP-2 portions have a high hydrophobic moment common to membrane-lytic peptides [5-9].

The transmembrane domain of gp41 was first deduced from the hydropathy plot of Env as a hydrophobic domain [10]. This transmembrane domain, herein referred to as the membrane-spanning domain (MSD), is composed of 23 highly conserved amino acid residues corresponding to amino acid residues 684 to 706 in the HXB2 strain (Figure 1A, B). An in vitro translation study in the presence of microsomal membranes suggested that HIV-1 Env has one MSD [11], as predicted by the hydropathy plot. In that study, the C-terminus of
C Biomed Central

() 2010 Liu et al; licensee BioMed Central Ltd. This is an Open Access article distributed under the terms of the Creative Commons Attribution License (<url>http://creativecommons.org/licenses/by/2.0</url>), which permits unrestricted use, distribution, and reproduction in any medium, provided the original work is properly cited. 


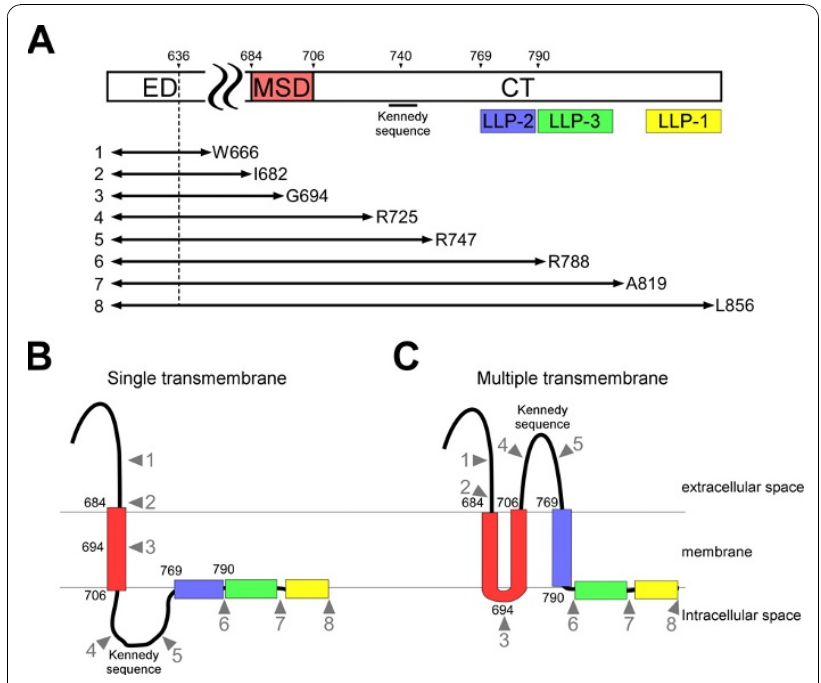

Figure 1 Schematic representation of Env mutants used in this study and the proposed topology models. (A) The points of truncation of gp41 were indicated together with a schematic diagram of the gp41 subunit. ED: ectodomain, MSD: membranespanning domain, CT: cytoplasmic tail, LLP: lentiviral lytic peptide. The numbering of the amino acid is based on that of the HXB2 strain. The vertical dashed line shows the position of the N-terminus of the gp41 used for the analysis in the bacterial system. The numbers and letters on the right indicate the position and the amino acid residue of the C-terminus. (B and C) Proposed topology models. The grey numbered arrowheads indicate the truncation points of gp41, the numbers and colors correspond to (A). gp41 was assigned to the cytoplasmic side of the cellular membrane [11], hence the gp41 subunit is regarded as a type I membrane protein with a single MSD. Other studies provided data consistent with this single MSD model. For example, two cysteine residues for palmitoylation are located in the cytoplasmic domain: one in the middle of LLP-1 (Cys-838) and the other at the upstream of LLP-2 (Cys-765) [12]. The internalization motif, YXXL (Tyr-769 to Leu-772), at the beginning of LLP-2 [13] also maps to the cytoplasmic domain of the single MSD model.

On the other hand, the mapping of the epitopes for neutralizing antibodies called into question the single MSD model. Some of the epitopes were mapped to the cytoplasmic region which contained the amino acid sequence known as the Kennedy sequence $\left({ }^{724}\right.$ PRGPD RPEGIEEEGGERDRDRS ${ }^{745}$ )[14-16] (Figure 1A). Furthermore, a report using an antibody raised against the LLP-2 portion revealed target binding during membrane fusion when added extracellularly [17]. As antibodies in general are not expected to cross intact membranes, an alternative membrane topology model of gp41 has been suggested in order to assign the mapped epitopes in the extracellular region [16] (Figure 1C). In this alternative model multiple MSDs were proposed because the C-terminus was assumed to be in the cytoplasm. Furthermore, the transmembrane portion of the single MSD model is expected to cross the membrane twice and one of LLPs, LLP2, is a putative third MSD (Figure 1C).

Several studies of the transmembrane portion of the single MSD model showed that it plays a critical role in the modulation of the membrane fusion process, which is an essential step of the HIV-1 life cycle [18-24]. Therefore analysis of the topology and structures of the transmembrane domain of gp41 is critical for our understanding of the mechanism of the membrane fusion. Furthermore the location of the neutralizing epitopes for antibodies is vital for a vaccine development.

In this study we reexamined gp41 topology in two different biological systems; prokaryotic and mammalian systems. The results of prokaryotic and mammalian systems without membrane fusion supported the single MSD model. The results obtained in the mammalian system in the presence of membrane fusion seem to support a transient alteration of the membrane topology of gp41. It is important, however, to note that the effect of the induction of membrane permeability during HIV1 Env-mediated membrane fusion cannot be excluded. The induction of membrane permeability was reduced by replacing the HIV-1 MSD with that of a foreign protein, CD22.

\section{Methods}

\section{Plasmid construction}

All PCR amplicons were first cloned into pCR4BluntTOPO using the TOPO cloning kit (Invitrogen, Carlsbad, CA) and sequences were verified.

For the topology analysis in the prokaryotic system, the expression vector pKMal-p2e was generated. pKMal-p2e has a kanamycin resistance gene derived from pK18 instead of $\beta$-lactamase in the context of pMal-p2e (NEB, Beverly, MA). The oligonucleotide adaptor generated by annealing the following two oligonucleotides: 5'-GTACCG AACAAT TACAC AAGCTTC GGATC CTCTAGA GTCGAC CTGCAG GC G-3' and 5'-AGCTC GC CTGCAG GTCGAC TCTAGA GGATCC GAAGCT TGTGTA ATTGTT CG -3' were inserted into pKMal-p2e to modify the multiple cloning site. This modified vector was named as mpKMal-p2e. The green fluorescent protein (GFP) gene as the reporter for the membrane topology was prepared by PCR using GFPopt ${ }_{1-11}$ in pCR4Blunt-TOPO [25] as the template with 5'-GAC TCTAGA ATGGTG AGCAAG GGCGAG GAGC-3' and 5'-GCACTG CAGTCA GGTGAT GCCGGC GGCGT-3' as the forward and reverse primer, respectively, and cloned into mpKMal-p2e vector using $X b a \mathrm{I}$ and Pst $\mathrm{I}$ sites. The generated vector was named as mpKMalp2e-GFP (Table 1). 
Table 1 Plasmids used in this study

\begin{tabular}{|c|c|}
\hline Plasmids & Description \\
\hline \multicolumn{2}{|l|}{ For prokaryotic system } \\
\hline mpKMalp2e-GFP & Multiple cloning site-modified pMalp2e containing $\mathrm{Kan}^{R}$ and Green fluorescent protein genes \\
\hline mpKMalp2e-gp41-1-GFP & mpKMalp2e-GFP with C-terminally truncated gp41 at W666 \\
\hline mpKMalp2e-gp41-2-GFP & mpKMalp2e-GFP with C-terminally truncated gp41 at 1682 \\
\hline mpKMalp2e-gp41-3-GFP & mpKMalp2e-GFP with C-terminally truncated gp41 at G694 \\
\hline mpKMalp2e-gp41-4-GFP & mpKMalp2e-GFP with C-terminally truncated gp41 at R725 \\
\hline mpKMalp2e-gp41-5-GFP & mpKMalp2e-GFP with C-terminally truncated gp41 at R747 \\
\hline mpKMalp2e-gp41-6-GFP & mpKMalp2e-GFP with C-terminally truncated gp41 at R788 \\
\hline mpKMalp2e-gp41-7-GFP & mpKMalp2e-GFP with C-terminally truncated gp41 at A819 \\
\hline mpKMalp2e-gp41-8-GFP & mpKMalp2e-GFP with full-length gp41 \\
\hline optGFP $_{1-11} /$ pET-47md & Modified pET-47b with modified super folder GFP \\
\hline \multicolumn{2}{|l|}{ For mammalian system } \\
\hline pHIVenv-Halo & The CMV promoter driven mammalian expression vector containing HaloTag gene \\
\hline pHIVenv-gp41-4-Halo & pHIVenv-Halo containing Env with C-terminally truncated gp41 at R725 \\
\hline pHIVenv-gp41-5-Halo & pHIVenv-Halo containing Env with C-terminally truncated gp41 at R747 \\
\hline pHIVenv-gp41-6-Halo & pHIVenv-Halo containing Env with C-terminally truncated gp41 at R788 \\
\hline pHIVenv-gp41-7-Halo & pHIVenv-Halo containing Env with C-terminally truncated gp41 at A819 \\
\hline pHIVenv-gp41-8-Halo & pHIVenv-Halo containing full-length Env \\
\hline pHIVenv-gp41-5 & Halo-deleted pHIVenv-gp41-5-Halo \\
\hline pHIVenv-gp41-8 & Halo-deleted pHIVenv-gp41-8-Halo \\
\hline pHIVenv-CD22-gp41-5 & The gp41 MSD replaced pHIVenv-gp41-5 with the MSD of CD22 \\
\hline pHIVenv-CD22-gp41-8 & The gp41 MSD replaced pHIVenv-gp41-8 with the MSD of CD22 \\
\hline pHook-Halo-GPI & The expression vector of the GPI anchored-HaloTag \\
\hline pKcTac-Halo & The expression vector of Tac antigen of IL-2 receptor fused with C-terminal HaloTag \\
\hline pKcTac-FLAG & pKcTacHalo vector whose HaloTag was replaced with 3xFLAG \\
\hline
\end{tabular}

This plasmid was used for the negative control for the experiment.

The near full-length gp41 gene derived from the HIV-1 HXB2 strain was amplified by PCR using pGEM7zf(+)-NB [23] as a template with 535fACC651 (Met):5'-AGTGGT ACCGAT GACGCT GACGGT ACAGGC CAGA-3' and 856 rXbaI: 5'-GTCTCT AGATAG CAAAAT CCTTTC CAAGCC CTG-3' as the forward and the reverse primer, respectively. The plasmid that harbors near full-length gp41 in pCR4blunt-TOPO was named as pEnv-HXb2gp41. For the construction of the gp41 mutants, the C-termini were serially truncated, (see Table 1 and Figure 1A), the various gp41 fragments were amplified by PCR using pEnv-HXb2gp41 as a template, with the oligonucleotide 535fACC651 as a forward primer, and the corresponding reverse primer designed for each truncation site. These truncated gp41 fragments were cloned into the vector mpKMalp2e-GFP with Hin$d$ III, which is present in the gp41 gene, and $X b a \mathrm{I}$ at the 5 ' and 3' terminus, respectively of the fragments. Figure 2A shows the resulting mpKMalp2e-gp41-GFP fusion constructs. The plasmid, optGFP ${ }_{1-11} / \mathrm{pET}-47 \mathrm{md}$ [26]
A

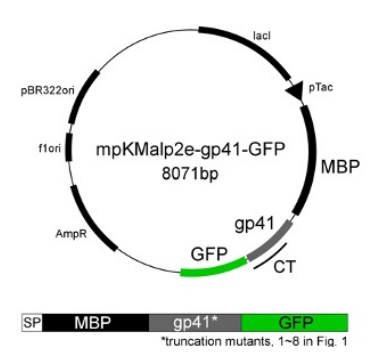

B

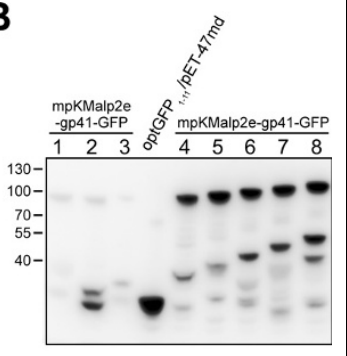

Figure 2 Constructs used to express recombinant gp41 in the E. coli system. (A) The expression vectors used in this study. The env gene is derived from HIV-1 HXB2 strain. gp41 starts amino acid 636 and ends with the various C-terminal positions as indicated in Fig. 1A. The schema below the plasmid map shows the components of the recombinant proteins. The truncated gp41 is preceded by maltose binding protein (MBP) and followed by the topological reporter, green fluorescent protein (GFP). The nomenclatures are as follows: lacl, lacl repressor gene; pTac, a hybrid promoter between trp and lac promoters; CT, cytoplasmic tail; AmpR, ampicilin resistant gene; f1ori, replication origin of $\mathrm{f1}$; pER322ori, replication origin of pBR322 plasmid. (B) Expression of the recombinant proteins. The immunoblotting of bacterial lysates probed with the anti-GFP antibody is shown. The name on the top of each lane indicates the expression vector used (see Table 1). 
that expresses GFP in the cytoplasm was used as a positive control.

The Halo7 gene was amplified by PCR using pFC14kHaloTag7 (Promega, Madison, WI) as a template, with 5'- GTCGAC GGCGGT GGCGGT AGCGGA TCCGAA ATCGGT ACTG-3' and 5'- GGTACC TTAACC GGAAAT CTCCAG AG -3' oligonucleotides as the forward and the reverse primer, respectively. The forward primer contained a SalI site and short linker sequence, $\mathrm{Gly}_{4} \mathrm{Ser}$, between the SalI site and Halo7 coding region. The reverse primer included an Acc65I site. The amplicon was inserted into the pHIVenvOPT vector, containing an envelope gene based on HXB2 strain that was optimized for human codon usage. The vector generated was named as pHIVenv-Halo (Figure 3A). To construct the truncation gp41 mutants for the mammalian analyses, five different positions were chosen as the

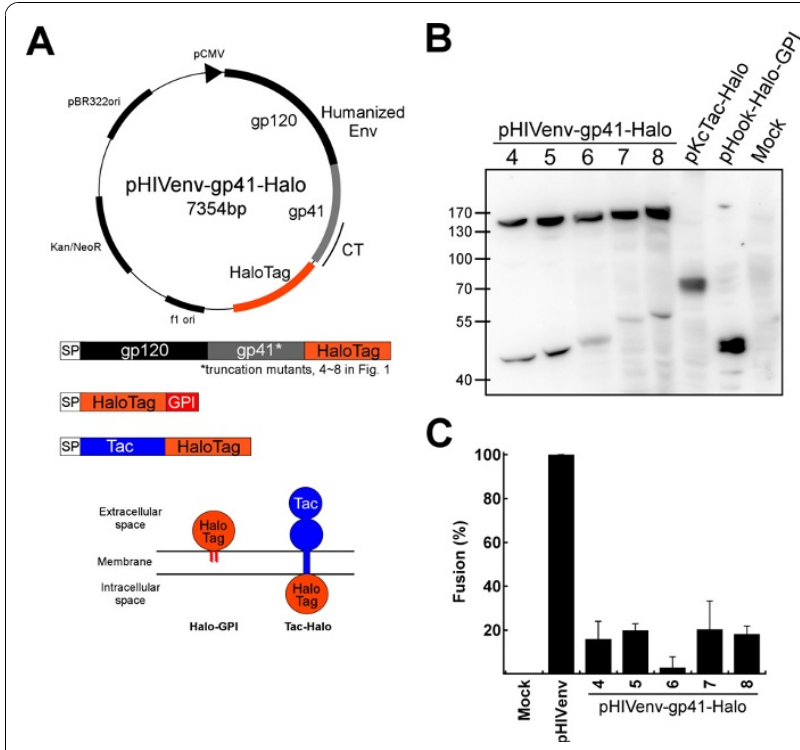

Figure 3 Constructs used for the expression of reporter proteins in the mammalian system. (A) The expression vector used in this study. The env gene of HXB2 origin was codonoptimized for human genes. The nomenclatures are as follows: pCMV, cytomegalovirus promoter; CT, cytoplasmic tail; HaloTag, Halo7 gene; f1 ori, replication origin of f1; Kan/NeoR, kanamycin or neomycin resistant gene; pER322ori, replication origin of pBR322 plasmid. The composition of the fusion protein used in the study was indicated below the plasmid map. The gp41 proteins with different C-terminal truncation points were fused to HaloTag at their C-terminus. The Halo-GPI, and Tac-Halo constructs and their expected membrane topology are shown schematically. (B) The result of immunoblotting with anti-HaloTag antibody. The names of the mammalian expression vector used are indicated above each lane. (C) Analysis of membrane fusion efficiency. The fusion activity of Halo-fused Env was evaluated by the syncytia-forming activity in 293CD4 cells. The percentage of the number of the nuclei included in syncytia was calculated by counting 300 nuclei in total. The constructs tested are indicated at the bottom of each bar; the number indicated the truncation points shown in Fig.1A.
C-terminal truncation points (Figure 1A and Table 1). The fragments of truncated env from XmnI to each termination codon were amplified by PCR using pHIVenvOPT as a template with 5'-GCTAGC AAATTA AGAGAAC-3' including the Sall site as the forward primer and the corresponding oligonucleotides at the truncated sites as the reverse primers, respectively. The env fragments were inserted into pHIVenv-Halo (Table 1). For the construction of pHIVenv-gp41-5 and pHIVenvgp41-8, stop codon-containing oligonucleotides generated by annealing 5'-TCGACTGATGAG -3' with 5'GTACCTCATCAG-3' was replaced with HaloTag gene to delete HaloTag. The Env expression vector with the MSD of CD22 [27] was constructed using PCR and replacement of the original MSD with the MSD of CD22. As for the control plasmids, two other expression vectors were constructed. The glycosylphosphatidylinositol (GPI)-anchored HaloTag gene was constructed as a marker for surface expression of HaloTag (Halo-GPI in Figure $3 \mathrm{~A}$ and Table 1). The GPI signal is derived from decay accelerating factor of human origin [28]. A Tac antigen, which is alpha subunit of Interleukin-2 receptor and is a single transmembrane protein [29], was fused with HaloTag gene at the C-terminus (Tac-Halo in Figure 3A and Table 1). This construct was used for the expression of the HaloTag protein in the cytoplasm. A derivative of this expression vector for Tac with a FLAG epitope at its C-terminus (Tac-FLAG) was generated by replacing the HaloTag sequence with that for 3xFLAG tag.

Expression of GFP-fused gp41 proteins and measurement of GFP fluorescence intensity

E. coli strain BL21 transformed with mpKMal-p2e carrying serially truncated gp 41 genes fused to GFP reporter was grown overnight at $22^{\circ} \mathrm{C}$ in TAG medium $(10 \mathrm{~g} / \mathrm{L}$ Tryptone, $5 \mathrm{~g} / \mathrm{L} \mathrm{NaCl}, 5 \mathrm{~g} / \mathrm{L}$ Glucose, $7 \mathrm{~g} / \mathrm{L} \mathrm{K}_{2} \mathrm{HPO}_{4}, 3 \mathrm{~g} /$ $\mathrm{L} \mathrm{KH}_{2} \mathrm{PO}_{4}, 1 \mathrm{~g} / \mathrm{L}\left(\mathrm{NH}_{4}\right)_{2} \mathrm{SO}_{4}, 0.47 \mathrm{~g} / \mathrm{L}$ Sodium Citrate) with $50 \mu \mathrm{g} / \mathrm{ml}$ kanamycin. The overnight bacterial culture was diluted 1:50 in $4 \mathrm{ml}$ TAG fresh medium containing $50 \mu \mathrm{g} / \mathrm{ml}$ kanamycin and growth was continued at $22^{\circ} \mathrm{C}$ until the $\mathrm{OD}_{600}$ reached 0.2 . Cells were grown for overnight in the presence of $0.1 \mathrm{mM}$ IPTG. Subsequently, one $\mathrm{ml}$ aliquot of culture was collected and resuspended in $0.5 \mathrm{ml}$ of PBS buffer and the GFP fluorescence intensity was measured by flow cytometry using a FACS Calibur (BD Biosciences, Mississauga, ON). At the same time, another $1 \mathrm{ml}$ aliquot of culture was dispensed for SDS-PAGE and immunoblotting analysis.

\section{Mammalian cell culture, transfection, labeling, and imaging}

The 293FT cells (Invitrogen, Carlsbad, USA) or 293CD4 cells (293 cells constitutively expressing human CD4) [23] 
were grown in 96-well Matriplates (GE Healthcare, Piscataway, NJ) with Dulbecco's modified Eagle medium (DMEM; Sigma, St. Louis, USA) supplemented with $10 \%$ FBS (Hyclone Labs., Logan, UT). In the case of 293FT, $5 \mu \mathrm{g} / \mathrm{ml}$ Geneticine (GiBco, Grand Island, USA) was further supplied. Cells were grown at $37^{\circ} \mathrm{C}$ in $5 \% \mathrm{CO}_{2}$ incubator.

DNA transfection of mammalian cells was performed using Fugene HD (Roche, Indianapolis, USA; Fugene HD $(\mu \mathrm{l}): \operatorname{DNA}(\mu \mathrm{g}): \operatorname{DMEM}(\mu \mathrm{l})=5: 2: 200)$. The transfection mix was incubated for 15 mins at room temperature prior to addition to the cell culture in a drop-wise manner $(10 \mu \mathrm{l}$ per well). After certain hours of transfection the transfected cells were subjected for further analyses as described below.

At the indicated time after transfection, the transfected cells were probed with HaloTag ligands. The starting time point of labeling after transfection was different for different experiments involving a different set of cells and vectors (see the Results section). The labeling was performed as suggested by the manufacturer (Promega). Briefly, the transfected live cells were labeled for 15 mins at $37^{\circ} \mathrm{C}$ with $1 \mu \mathrm{M}$ of HaloTag ligand Alexa Fluor 488 (AF488), a membrane-impermeable ligand, or Oregon Green (OG), a membrane permeable ligand, respectively. After labeling, the cells were rinsed three times with 200 $\mu \mathrm{l}$ prewarmed DMEM plus 10\% FBS and subsequently incubated at $37^{\circ} \mathrm{C}$ with $5 \% \mathrm{CO}_{2}$ for 30 mins. The medium was changed with fresh warm DMEM plus $10 \%$ FBS, then images were captured using a confocal microscope (Olympus FluoView FV1000, Tokyo, Japan).

Immunofluorescent staining assay using the anti-FLAG monoclonal antibody (Sigma) was performed to detect the FLAG-tagged proteins as below. Following the fixation of the transfected cells with $2 \%$ paraformaldehyde at $25^{\circ} \mathrm{C}$ for 5 mins, the anti-FLAG antibody $(1 / 200$ in $0.5 \%$ BSA and PBS) was used as the first antibody. After incubating at room temperature for $1 \mathrm{~h}$, the cells were rinsed 3 times with $200 \mu \mathrm{l}$ prewarmed PBS plus $0.5 \%$ BSA and subsequently incubated with anti-mouse antibody conjugated with AlexaFluor 488 (Invitrogen) $(1 / 200$ in $0.5 \%$ BSA and PBS) at room temperature for $1 \mathrm{~h}$. The images were captured using a confocal microscope (Olympus).

To evaluate the cell viability, staining with propidium iodide (PI) [30] was used. In the case of co-labeling with the HaloTag ligands, staining with AF488 was performed first, then PI staining for $15 \mathrm{~min}$ at room temperature with a final concentration of $2.5 \mu \mathrm{g} / \mathrm{ml}$ followed. The cells were rinsed two times with PBS and images were analyzed as described above. In the case of costaining with anti-FLAG monoclonal antibodies, PI staining was performed first, followed by labeling with the anti-FLAG monoclonal antibody.

To mimic the effect of the conformational changes of gp120 after its binding to the CD4 receptor, soluble
CD4 was added to the 293FT cells transfected with HIV-1 Env expression vectors. The soluble CD4 protein (final concentration: $0.1 \mu \mathrm{M}$ ) was kept in the medium since immediately after transfection.

\section{Syncytia formation assay}

A syncytia formation assay was performed by transfecting the HIV-1 Env expression vectors (listed as For mammalian system in Table 1) into the 293CD4 cells. The cells were transfected when they were about $50 \%$ confluent. At $48 \mathrm{~h}$ after transfection, the images were captured with IN Cell analyzer 1000 (GE Healthcare, Uppsala, Sweden). The fusion activity of Halo-fused Env was evaluated by counting 300 nuclei in total after staining with $2 \mu \mathrm{M}$ Hoechst and determining the percentage of nuclei included in syncytia.

\section{Immunoblot analysis}

Bacterial cultures $(1 \mathrm{ml})$ were harvested and resuspended in $50 \mu \mathrm{l}$ SDS-PAGE loading buffer (2\% SDS, 2 mM DTT, 10\% glycerol, $50 \mathrm{mM}$ Tris- $\mathrm{HCl}, \mathrm{pH} 6.8,0.01 \%$ Bromo phenol blue). The mixture was kept for 10 mins at $95^{\circ} \mathrm{C}$ and subjected to centrifugation $\left(20,000 \mathrm{~g}, 4^{\circ} \mathrm{C}\right)$ with MX-301 (Tomy, Japan) to remove the pellets. Whole cell lysates $(2 \mu \mathrm{l})$ were resolved using a $5-20 \%$ gradient SDS polyacrylamide gel (DRC, Tokyo, Japan). The proteins were transferred to the PVDF membrane and probed with 15,000-fold diluted anti-GFP antibody (Santa Cruz Biotechnology, Santa Cruz, USA) for $1 \mathrm{~h}$ at room temperature. Anti-mouse antibody (GE healthcare), diluted by 5,000 -fold, was used as the secondary antibody. The signal was developed by streptavidinbiotinylated horseradish peroxidase complex (GE healthcare) and the chemiluminescence reagents (Roche), and detected by LAS3000 (Fuji).

The transfected 293FT cells grown in 10-cm dishes as described above were collected and centrifugated $(5,000$ $\mathrm{g}, 4^{\circ} \mathrm{C}$ ) with MX-301. The cell pellet was lysed with $250 \mu$ of RIPA lysis buffer [ $50 \mathrm{mM}$ Tris- $\mathrm{Cl}$ (pH 7.4), 150 $\mathrm{mM} \mathrm{NaCl}, 1 \% \mathrm{NP}-40,0.1 \% \mathrm{SDS}]$ and then centrifuged (MLA-130 rotor, 100,000rpm, 30 mins, $4^{\circ} \mathrm{C}$ ) with Beckman Optima ${ }^{\mathrm{Tm}}$ Max Ultracentrifuge. The supernatant (20 $\mu \mathrm{l})$ was treated with the same method as described above. The protein bands on the PVDF membrane were developed as described above, except for the 500-fold diluted anti-Halo pAb (Promega) and 5000-fold diluted anti-rabbit antibody (GE healthcare) which were used as the primary and secondary antibodies, respectively.

\section{Results}

Topology mapping of gp41 using GFP as a reporter in a prokaryotic system

We first employed the well-established prokaryotic topological analysis using GFP as a reporter [31,32]. If 
GFP is located in the cytoplasm it folds into an active form, whereas when it is translocated into the periplasm it is non-functional [31]. The periplasm-targeted maltose-binding protein was placed at the $\mathrm{N}$-terminus of the gp41 portion to be tested, and then GFP, a topological reporter, was fused to the C-terminus of the gp41 fragment (Figure 2A). The series of gp41 proteins truncated at the different $\mathrm{C}$-terminal positions were tested (Figure 1A and Table 1). The $\mathrm{N}$-terminus of gp41 portion included was fixed at the position of 636th amino acid close to the predicted MSD (Figure 1A dotted line), because there is little controversy on the beginning of the MSD itself.

After transformation of E. coli. with one of the plasmids, the expression of the recombinant protein was evaluated by immunoblotting using an anti-GFP antibody and the results are shown in Figure 2B. The levels of protein expression with mpKMalp2e-gp41-1-GFP, mpKMalp2e-gp41-2-GFP, and mpKMalp2e-gp41-3-GFP, were low (Figure 2B), and we did not analyze these constructs further. The rest of constructs each expressed a comparable amount of the fusion protein of about $100 \mathrm{kD}$ (Figure 2B). The fluorescence intensities of GFP at $530 \mathrm{~nm}$ of $E$. coli induced for the expression of the fusion proteins were measured by a flow cytometry. Compared with the negative control that expresses GFP in the periplasm (mpKmalp2e-GFP), the GFP intensity adjusted by the cell density was significantly higher for mpKMalp2e-gp41-4-GFP, mpKMalp2e-gp41-5-GFP, mpKMalp2e-gp41-6-GFP mpKMalp2e-gp41-7-GFP and mpKMalp2e-gp41-8-GFP (Table 2). This suggested that GFP attached at the position 4 to 8 lies in the cytoplasm. Interestingly, there was no significant difference in the GFP fluorescent intensity adjusted by the level of the expression for mpKMalp2e-gp41-4-GFP, mpKMalp2e-gp41-5-GFP, mpKMalp2e-gp41-6-GFP, mpKMalp2e-gp41-7-GFP and mpKMalp2e-gp41-8-GFP. These data suggested that there was no topological shift of GFP reporter in these regions; therefore the Kennedy sequence and LLP regions are not exposed to the

\section{Table 2 Results of GFP quantification}

\begin{tabular}{ll}
\hline Vector & $\begin{array}{l}\text { Adjusted GFP signal } \\
\text { (The number of counts } \\
\text { /OD }_{\mathbf{6 0 0}} \text { ) }\end{array}$ \\
\hline optGFP $_{1-11} /$ pET-47md & 4026.238 \\
\hline mpKMalp2e-gp41-4-GFP & 1103.775 \\
\hline mpKMalp2e-gp41-5-GFP & 971.453 \\
\hline mpKMalp2e-gp41-6-GFP & 828.177 \\
\hline mpKMalp2e-gp41-7-GFP & 1018.790 \\
\hline mpKMalp2e-gp41-8-GFP & 986.997 \\
\hline mpKmalp2e-GFP & 313.958 \\
\hline
\end{tabular}

periplasmic region. These results are consistent with the single MSD model of gp41 (Figure 1B).

\section{Expression of HaloTag-attached HIV-1 Env in mammalian cells}

Although the bacterial system is quick and informative, eukaryote specific post-translational modifications and/ or the difference in the composition of lipids in the membrane may affect the topology of gp41. Therefore, HIV-1 Env with the C-terminus of gp41 linked to HaloTag was expressed in mammalian cells (Figure 3A). The HaloTag is a $33 \mathrm{kDa}$ protein designed to covalently bind to its membrane-permeable/impermeable ligands conjugated with a fluorescent chromophore [33]. Based on the previous published results [11] and our own results of the prokaryotic system (see above), we focused on the analysis of the region after the predicted MSD of the single MSD model (truncation positions 4-8 in Figure 1A). The GPI-anchored HaloTag protein (Halo-GPI) and the HaloTag attached to the C-terminus of the Tac antigen after MSD (Tac-Halo) were made as the controls for the extracellular and intracellular positioning of HaloTags, respectively (Figure 3A and Table 1). Expression of HaloTag-attached envelope proteins was confirmed by immunofluorescence analysis with anti-gp120 antibody (data not shown) and immunoblotting analysis with anti-Halo antibodies (Figure 3B). The bands around 130-170kD and 40-55kD for pHIVenv-gp41-Halo are HaloTag-attached gp160 and gp41, respectively.

The membrane fusion capacity of these mutants was examined with a syncytia formation assay by transfecting the expression vector into 293CD4 cells [23]. Although the efficiency of the fusion was reduced in all of the HaloTag-attached envelope proteins, all still retained membrane fusion activity (Figure $3 \mathrm{C}$ ). When we analyzed the fusion activity with the DSP assay [34], better fusion was observed (data not shown). Since the DSP assay relies on the smaller reporter proteins, the presence of the defect of pore dilatation in HaloTag attached mutants was suggested.

\section{Topology mapping of gp41 in mammalian cells using HaloTag-specific membrane-impermeable ligands}

The membrane-permeable and membrane-impermeable ligands with fluorescent chromophore available for HaloTag were used to examine the location of the attached HaloTag in relation to the cell membrane. Oregon Green (OG) that readily cross the cell membrane labels HaloTag located in both extracellular and intracellular spaces, whereas Alexa Fluor 488 (AF488), a membrane-impermeable ligand, should label HaloTag exposed on the cell surface. When we used the membrane-permeable substrate, OG, all of the 293FT cells transfected with HaloTag-fused truncated Env 
plasmids (pHIVenv-gp41-4-Halo, pHIVenv-gp41-5Halo, pHIVenv-gp41-6-Halo, pHIVenv-gp41-7-Halo, and pHIVenv-gp41-8-Halo) were stained by the ligand (Additional File 1; Figure S1). The 293FT cells transfected with pHook-Halo-GPI and pKcTac-Halo were also stained by OG; the fluorescent signal was localized at the rim of the cells (Additional File 1; Figure $\mathrm{S} 1$ ). On the other hand, when we used the membraneimpermeable substrate, AF488, none of the 293FT cells transfected with the plasmids harboring HaloTag-fused Env with C-terminal truncation were stained (Figure 4 pHIVenv-gp41-4 to -8-Halo). As expected, the 293FT cells transfected with pHookHalo-GPI were stained by AF488, but the 293FT cells transfected with pKcTac-Halo did not show any fluorescent signal under the same labeling and imaging conditions (Figure 4), verifying the authenticity of this experimental system. These results indicate that the HaloTag attached at positions 4 to 8 of gp 41 are located in the cytoplasm of the cells. This result is consistent with the prokaryotic data (Table 2) and suggests that Kennedy sequence and LLP regions are both located in the cytoplasm, supporting the single MSD model of gp41 [11].

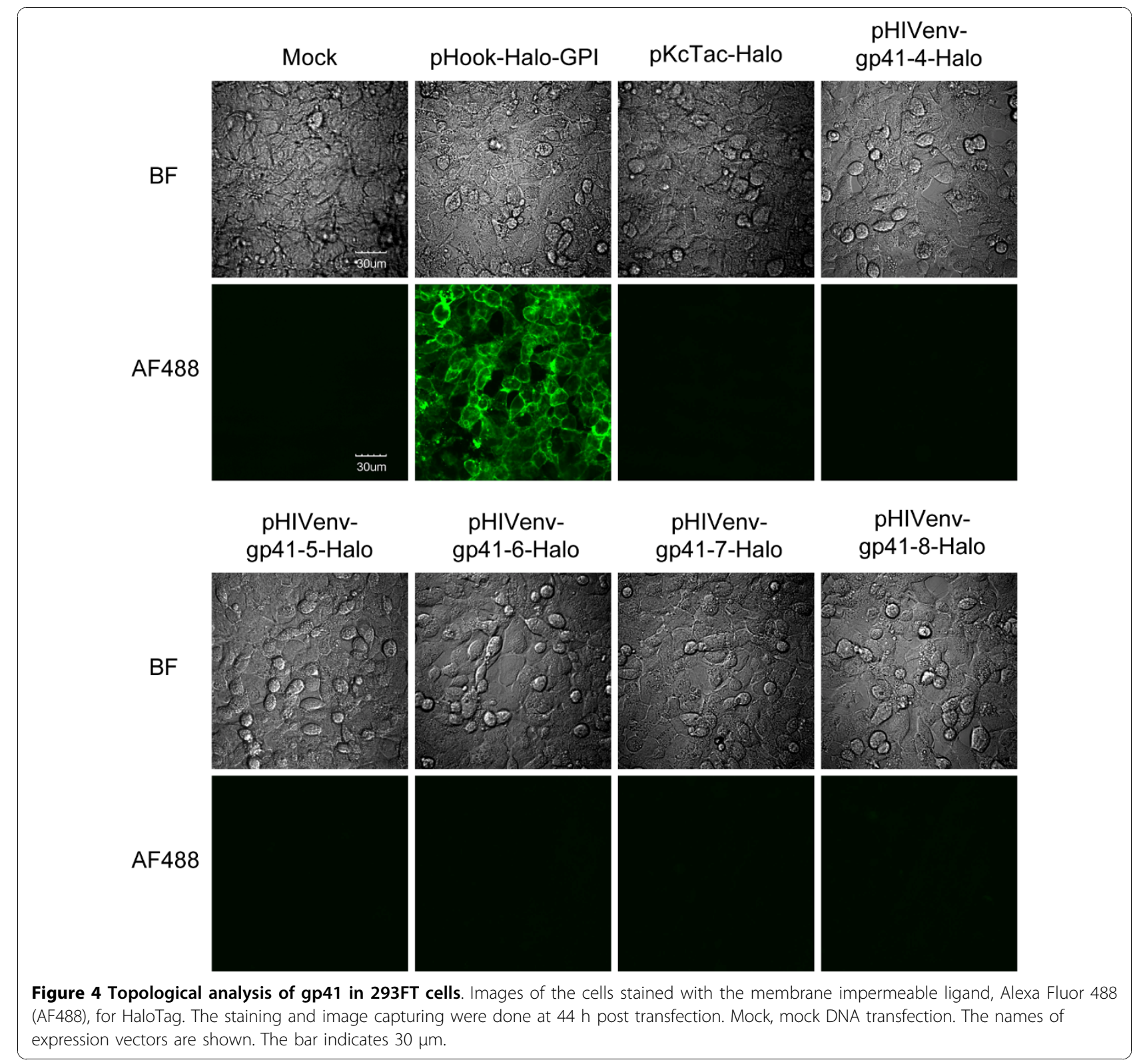




\section{Examination of membrane topology with HaloTag in syncytia formed in 293CD4}

As the possibility for a transient topological change of gp41 during membrane fusion has been proposed $[16,17]$, we induced the formation of syncytia in 293CD4 by transfecting a series of Env-HaloTag expression vectors and performed the labeling. All of the syncytia formed after transfecting the expression vector for each Env-HaloTag were positively stained with OG, membrane-permeable ligand, during membrane fusion, confirming the expression of Halo-fused Envs (Additional File 2; Figure S2). When the membrane-impermeable ligand AF488 was used for staining, most of the multinucleated 293CD4 cells expressing various gp41 truncation mutants were not stained (Figure 5). The only exception was the cells transfected with pHIVenvgp41-5-Halo, in which rare and weak staining of the syncytia were observed (Figure 5). Even the later time points with the similar levels of syncytia formation with pHIVenv-gp41-8-Halo were chosen to compensate the reduced fusion efficiency of pHIVenv-gp41-5-Halo, the staining incidence for pHIVenv-gp41-5-Halo did not increase. The 293CD4 cells transfected with the control plasmids, pHook-Halo-GPI (HaloTag on the cell surface) and pKcTac-Halo (HaloTag in the cytoplasm), showed the results consistent with their expected topological locations (Figure 5 pHook-Halo-GPI and pKcTac-Halo).

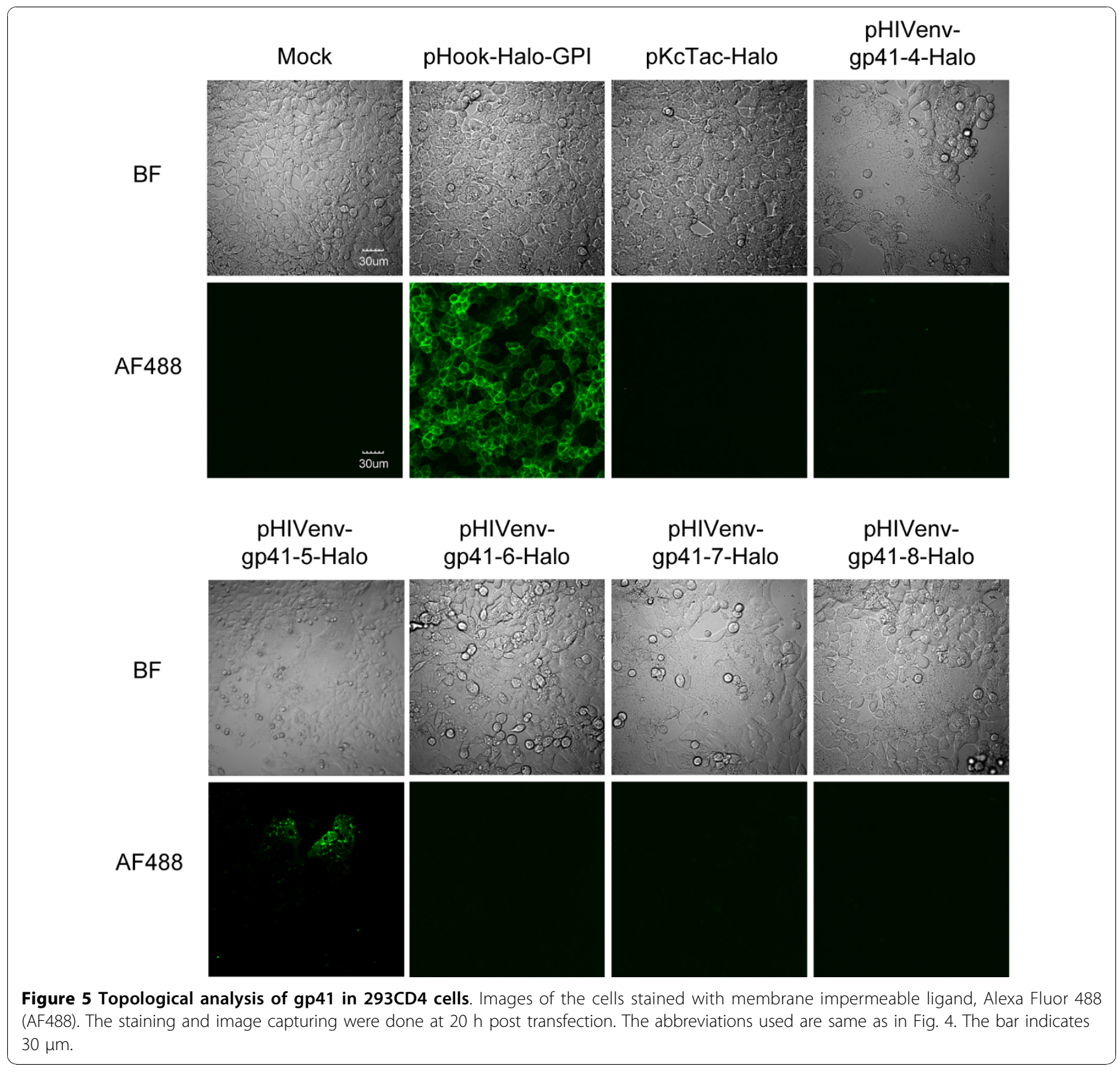


When the 293CD4 cells transfected with pHIVenv-gp415-Halo were stained with the anti-HaloTag antibody without permeabilization procedure, rare events of staining were observed (data not shown). These results suggest that the possibility of sporadic exposure of cytoplasmic domain of gp41 during membrane fusion with pHIVenv-gp41-5-Halo.

\section{Augmented membrane permeability by Env-induced membrane fusion}

The result shown above for pHIVenv-gp41-5-Halo could be an indication of a rare translocation of the cytoplasmic region of the gp41. The reason why the translocation, if happening, is limited to the truncation at position 5 with a very low incidence was not clear. Since there was no staining for pHIVenv-gp41-4-Halo, we have to assume a hypothetical MSD between the position 4 and 5 . This is to assume the Kennedy region to be the hypothetical MSD and is different from the model shown in Figure 1C. The hydrophilic Kennedy sequence is not likely to be an MSD by several prediction algorithms (Table 3). An alternative possibility is that the sporadic staining was due to the induced permeability of membranes in syncytia.

To distinguish the alteration of gp41 topology from membrane permeability induced during membrane fusion, we co-expressed tag-free HIV-1 Env together with Tac-Halo in the same cells. Namely, the pKcTacHalo, and pHIVenv-gp41-5/pHIVenv-gp41-8 or pHIVenv-CD22-gp41-5/pHIVenv-CD22-gp41-8 (Table 1) were co-transfected simultaneously. We then probed the HaloTag expressed in the cytoplasmic side (see Figure 3A) with AF488, membrane-impermeable ligands. Both 293FT (fusion incompetent) and 293CD4 (fusion competent) cells were used to determine the effect of membrane fusion. The co-transfected 293FT cells were not stained with AF488 (Figure 6 -soluble CD4), whereas these cells were stained with OG (data not shown). The expressions of Env in 293FT cells were confirmed by immunoblotting (Additional file 3; Figure S3). The addition of soluble $\mathrm{CD} 4$, which can induce the early conformational change of gp120, did not show any changes in the staining patterns (Figure $6+$ soluble CD4).

\section{Table 3 Computational analyses of possible} transmembrane domain

\begin{tabular}{ll}
\hline Program & $\begin{array}{l}\text { Region of the predicted membrane-spanning segment } \\
\text { (original: 684-706) }\end{array}$ \\
\hline TroPred & $684-705$ \\
\hline TMHMM & $678-701$ \\
\hline SOSUI_MP1 & $675-708$ \\
\hline SOSUI & $683-706$ \\
\hline
\end{tabular}

In the case of 293CD4 cells, however, the co-transfected cells (Figure 7 -C34, pHIVenv-gp41-5 or $8+$ pKcTac-Halo,) could be clearly stained by AF488 at the site of syncytium (Figure $7-\mathrm{C} 34$ ). These staining were not due to the cell death, because some cells labeled with AF488 did not show the staining with propidium iodide (Figure 7 shown in red). The staining with AF488 was abolished when membrane fusion was inhibited by the addition of $\mathrm{C} 34$, an inhibitor of six-helix bundle formation (Figure $7+\mathrm{C} 34$, pHIVenv-gp41-5 or -8 + pKcTac-Halo). These results indicated that the induction of the permeability was dependent on active membrane fusion.

To examine whether the observed membrane permeability during membrane fusion allows antibodies to penetrate membranes, we probed the $3 \times$ FLAG epitope attached to the cytoplasmic portion of the Tac antigen (Tac-FLAG) with the anti-FLAG antibody. The intracellular $3 \times$ FLAG tag was detectable when HIV-1 Env with or without the truncation, pHIVenv-gp41-5 and pHIVenv-gp41-8, respectively, were co-expressed (Figure 8 -C34). Although the staining pattern of each syncytium varies, it seemed that the incidence of the positively stained syncytia was slightly lower than that obtained with the membrane-impermeable ligands shown in Figure 7. When the membrane was permeabilized with detergent prior to antibody staining, all of syncytia were stained well (data not shown). These results suggest both the full-length and truncated Env have the ability to permeabilize the membrane to allow the antibodies to cross the membranes.

\section{Augmented membrane permeability is dependent on MSD sequence}

Since membrane permeability was induced in the cells transfected with pHIVenv-gp41-5-Halo, the presence of LLPs is not required for the increased permeability. To further characterize the region required for this enhanced permeability we constructed the mutants in which the original gp41 MSD was replaced with the foreign MSD derived from CD22 [27] in the pHIVenv context. Previous reports indicated that the MSD derived from CD22 did not alter the function of HIV-1 Env [27]; however, the replacement seemed to delay the appearance of syncytia when compared with the wild type (see below). We compared these mutants with the HIV-1 Env with the native MSD. In the case of the HIV-1 Env with its native MSD, intracellular HaloTag was detectable with membrane-impermeable AF488 at the earlier time point after co-transfection $(16 \mathrm{~h}$ post transfection, Figure 7; pHIVenv- gp41-5 and 8). On the other hand, there was minimal staining in cells co-transfected with HIV-1 Env with CD22 MSD at $16 \mathrm{~h}$ after transfection (data not shown). At $44 \mathrm{~h}$ post transfection when the cells transfected with the native gp41 MSD were almost gone due to 


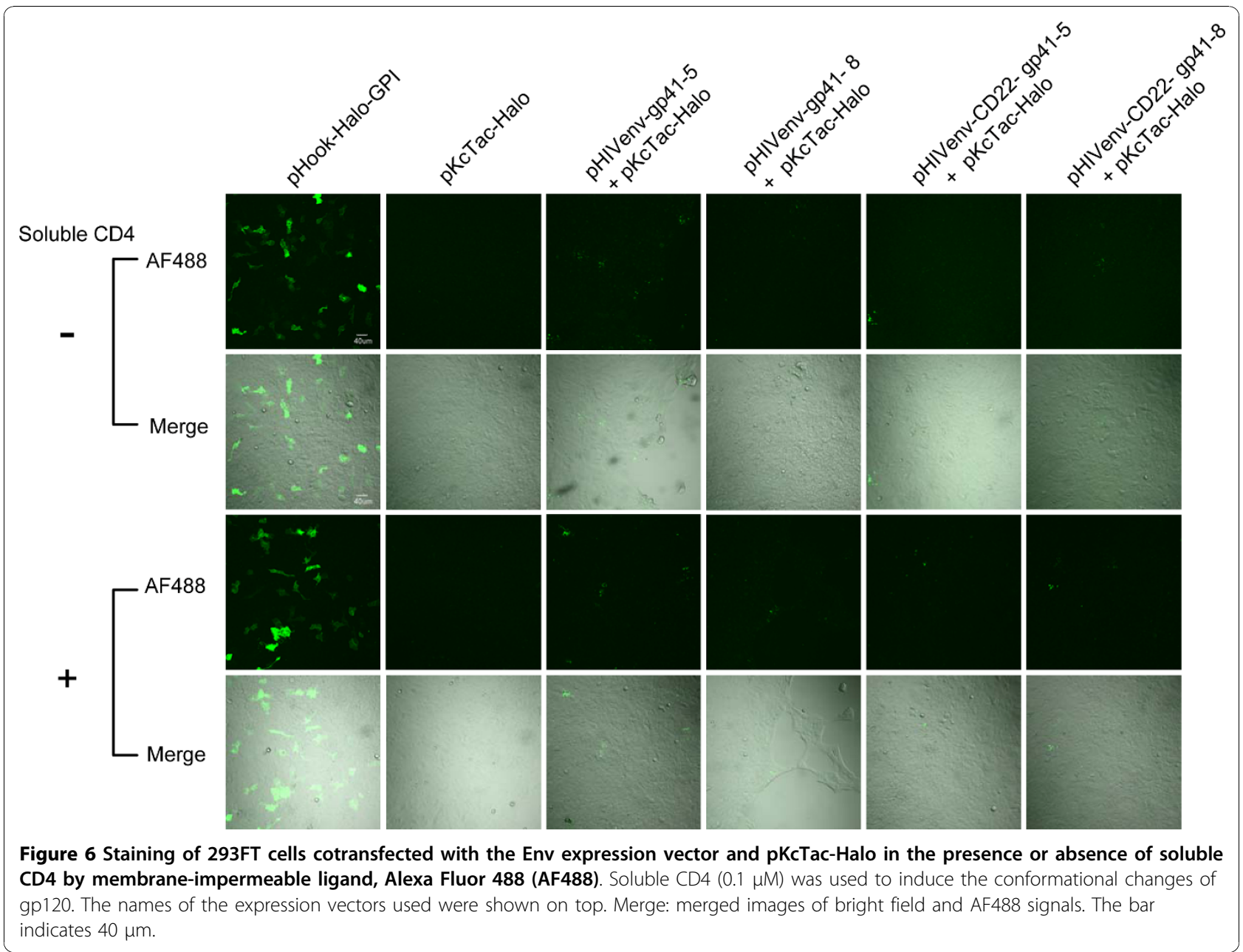

the cell death, some cells transfected with CD22 MSD mutants were stainable with AF488 (Figure 7 -C34, pHIVenv-CD22-gp41-5 or $8+$ pKcTac-Halo). Therefore there is a significant difference in the pattern of the staining between the native and CD22 MSDs. At $44 \mathrm{~h}$ post transfection, there were more dead cells as indicated by the positive PI staining. These cells were also stained with AF488. There are, however, some syncytia stained only with AF488 for CD22 MSD mutants (Figure 7). Inhibition of the membrane fusion with $\mathrm{C} 34$ blocked the staining (Figure 7 + C34). Similar results were obtained if anti-FLAG antibodies were used to detect the FLAG tag located in the cytoplasm. (Figure 8 pHIVenv-CD22-gp41-5 or $8+$ pKcTacFLAG). Taken together, these results indicated that the induction of permeability was membrane fusion-dependent and that the gp41 MSD played some role in the degree of induced permeabilization during membrane fusion.

\section{Discussion}

In this study we examined the membrane topology of the gp41 subunit in two different biological systems.
The truncated gp41 subunit was tagged with the topological reporter protein at the C-terminus (Figure 1, 2, 3). A prokaryotic reporter, GFP [31,32] and mammalian reporter, HaloTag [33], were used. Both reporters enabled us to examine the topology in living cells without the artifacts caused by fixing.

In our prokaryotic system, all of the tested constructs (mpKMalp2e-gp41-4, 5, 6, 7- and 8-GFP) showed stronger GFP fluorescence than the control. This suggested that gp41 had a single MSD that places the Kennedy sequence, LLP-2, LLP-3, LLP-1 and the C-terminus of gp41 in the cytoplasmic side. The analysis with $\beta$-lactamase, another topology reporter, which is only active in periplasm produced the data consistent with that of GFP (data not shown). These data are consistent with the results obtained by the currently available several programs for prediction of transmembrane domains (Table 3).

Our analysis of gp41 topology in mammalian cells without membrane fusion (293FT cells) supported the single transmembrane model, concordant with that of 


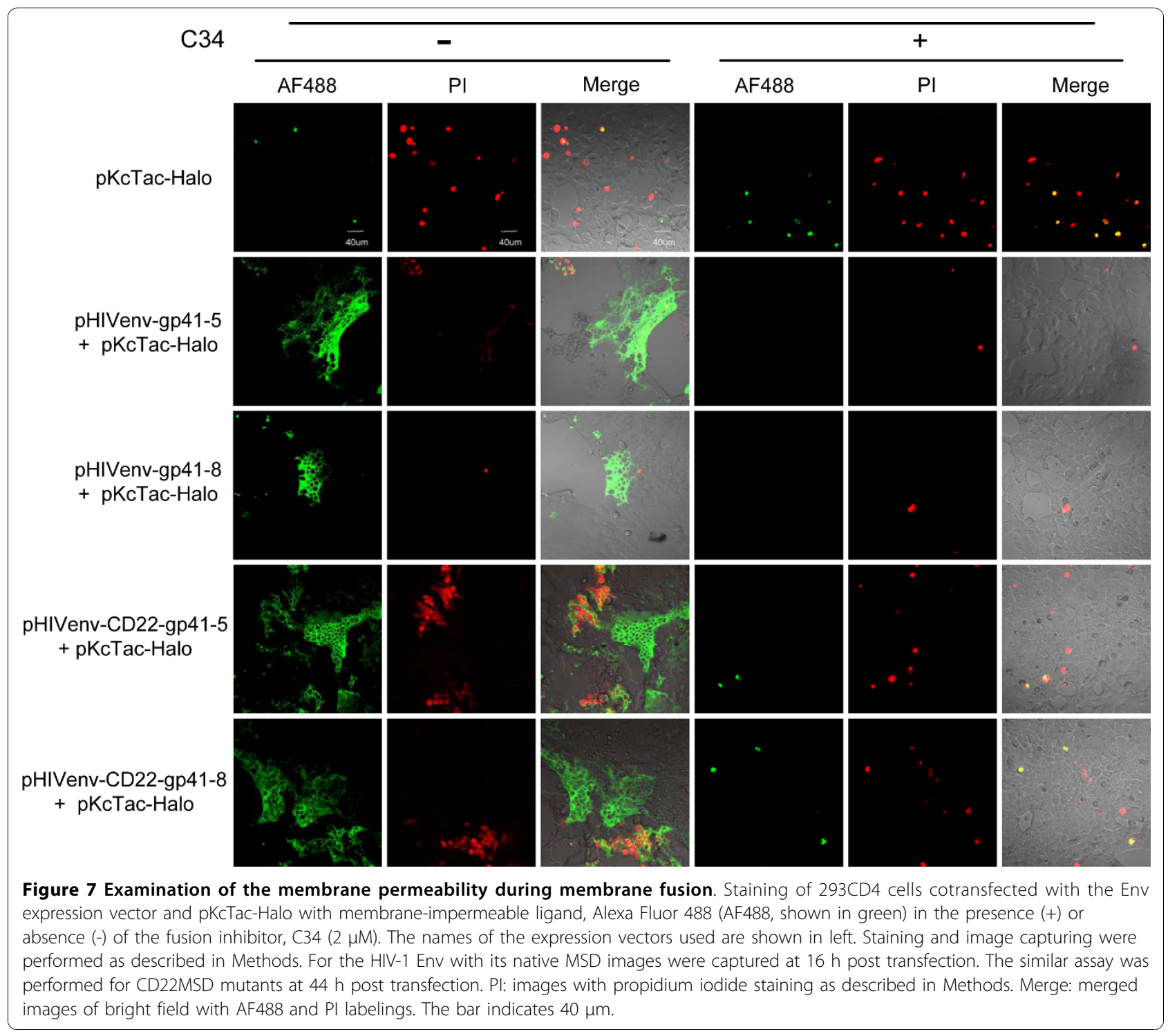

the prokaryotic system. Only sporadic staining with membrane impermeable ligands was observed for $\mathrm{pHI}$ Venv-gp41-5-Halo (truncation at position 747 in HXB2 Env) in fusion-competent 293CD4 cells. Since staining for the preceding truncation point, pHIVenv-gp41-4Halo (truncation at the residue 725 in $\mathrm{HXB} 2$ ), was negative, suggesting an additional MSD between the residue 725 and 747 . This region contains about 20 mainly hydrophilic amino acid residues that correspond to the Kennedy sequence. As shown in Table 3 no MSD was predicted in this region by several computational algorithms. So we speculated that there is other reason for the observed apparently contradictory observations.

We examined the possibility of enhanced membrane permeability to account for the staining of putative intracellular regions during membrane fusion. The intracellularly located HaloTag was labeled using the membrane-impermeable ligand for HaloTag when HIV1 Env-mediated membrane fusion occurred (Figure 7). Antibodies were also able to stain the intracellular targets in conditions of membrane fusion (Figure 8). Although we cannot completely exclude the possible alternations of the gp41 topology, the multiple MSD model based on the epitope mapping $[16,17]$ needs to be reevaluated carefully given the increased permeability we have observed.

We attempted to map the region(s) of gp41 responsible for increased membrane permeability. Our data suggested that the increased permeability was dependent on active membrane fusion (Figure 7 and 8). It is consistent with several reports of the fusion-dependent induction of membrane permeability in HIV-1 infected cells [35-38]. 


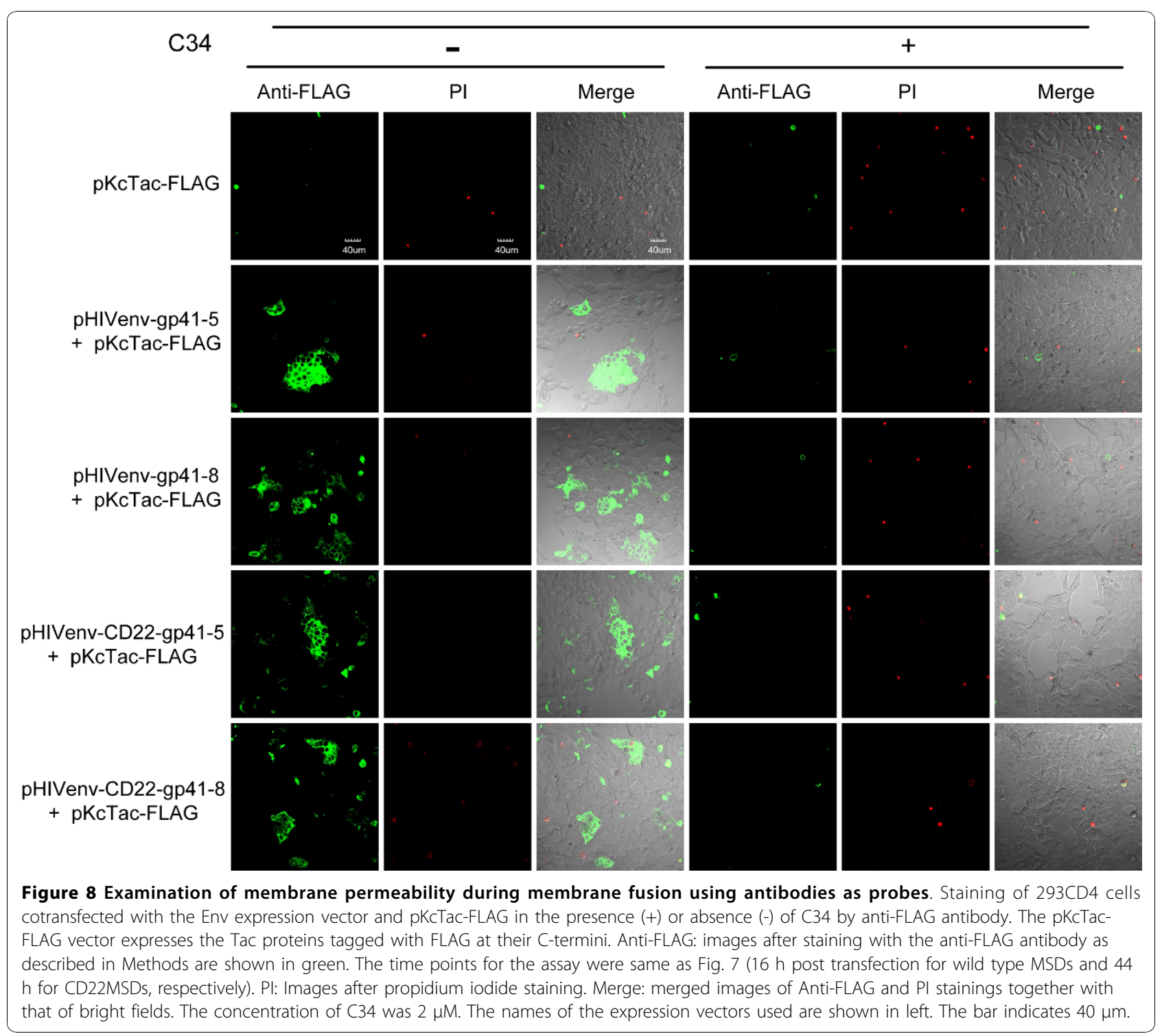

It is known that the several isolated subdomains of gp41, critical for membrane fusion, have potential to permeabilize the mammalian and the bacterial membranes [39-50]. These include the fusion peptide, the membrane proximate external region, the MSD and the LLPs. Our data of pHIVenv-gp41-5 (Figure 7 and 8) excludes the possibility of the role of LLPs. Our data using MSD replacement mutants pHIVenv-CD22-gp41-5 or pHIVenvCD22-gp41-8, suggested the MSD affected the level of permeability, but whether the MSD directly affected the permeability or the effect was mediated via the efficiency of the membrane fusion was hard to be determined.

Since our assay relied on the topological reporter proteins attached at the C-termini of truncated gp41 proteins, the possibility of artifacts cannot be excluded. The exact reason why neutralizing epitopes mapped to the cytoplasmic regions remains unclear. There are at least two possible explanations. First, it is possible that the some of the antibodies themselves are intrinsically membrane permeable. Second, permeability that is sufficient to permit antibodies to cross the membrane may be induced by membrane fusion. Although our findings support the latter model, further study is needed to explore whether an alternative topology for gp41 MSD during membrane fusion really takes place and if such a possibility is a general phenomenon for other HIV-1 strains.

\section{Conclusions}

The membrane topology of the gp41 subunit of HIV-1 Env was examined in both prokaryotic and mammalian systems. The topology with a single MSD was supported in both systems. In addition, augmented membrane 
permeability was shown to be dependent on both the sequence of MSD and active membrane fusion.

\section{List of abbreviations}

Env: envelope glycoprotein; HIV-1: human immunodeficiency virus type-1: LLP: lentiviral lytic peptide; MSD: membrane-spanning domain; GFP: green fluorescent protein; GPI: glycosylphosphatidylinositol; FBS: fetal bovine serum; OG: Oregon Green; AF488: Alexa Fluor 488; PI: propidium iodide; PBS: phosphate buffered saline.

\section{Acknowledgements}

This work was supported by a contract research fund from the Ministry of Education, Culture, Sports, Science and Technology for Program of Japan Initiative for Global Research Network on Infectious Diseases. We thank Dr. Kunito Yoshiike for his critical reading of the manuscript.

\section{Additional material}

Additional file 1: Supplemental Fig.1 Detection of HaloTag-attached HIV-1 Env in 293FT cells. Images of the transfected 293FT cells stained with membrane-permeable ligand, Oregon Green (OG). BF indicates the bright filed images. The names of the expression vectors are shown. Mock, mock DNA transfection.

Additional file 2: Supplemental Fig.2 Detection of HaloTag-attached HIV-1 Env in 293CD4 cells. Images of the transfected 293CD4 cells stained with membrane permeable ligand, Oregon Green (OG). The nomenclature used are same as Supple Figure 1.

Additional file 3: Supplemental Fig.3 Expression of HIV-1 Env with its native MSD or foreign CD22MSD in 293FT cells. The expression of the envelope protein was examined by immunoblotting using the antigp120 antibody as described previously [23]. The names of the expression vectors were shown on top. The bands of gp160 and gp120 are indicated.

\section{Author details}

'China-Japan Joint Laboratory of Structural Virology and Immunology, Institute of Biophysics, Chinese Academy of Sciences, 15 Datun Road, Chaoyang District, Beijing 100101, P. R. China. ${ }^{2}$ Research Center for Asian Infectious Diseases, and 3Division of Infectious Diseases, Advanced Clinical Research Center, Institute of Medical Science, University of Tokyo, 4-6-1, Shirokanedai Minato-ku, Tokyo 108-8639, Japan. ${ }^{3}$ Current Address: Department of Pediatrics, Emory University School of Medicine, 2015 uppergate Dr. Atlanta, GA 30322, USA.

\section{Authors' contributions}

SL, NK, YL and DX performed the experiments. The analysis in the prokaryotic system was done by SL and DX. The work in mammalian system was performed by SL, NK and YL. The study was conceived by ZM. Al supervised the entire work. SL, NK and ZM wrote the manuscript. All authors read and approved the final manuscript.

\section{Competing interests}

The authors declare that they have no competing interests.

Received: 13 July 2010 Accepted: 30 November 2010 Published: 30 November 2010

\section{References}

1. Dettenhofer M, Yu XF: Characterization of the biosynthesis of human immunodeficiency virus type $1 \mathrm{Env}$ from infected T-cells and the effects of glucose trimming of Env on virion infectivity. J Biol Chem 2001, 276:5985-5991.

2. Otteken A, Earl PL, Moss B: Folding, assembly, and intracellular trafficking of the human immunodeficiency virus type 1 envelope glycoprotein analyzed with monoclonal antibodies recognizing maturational intermediates. J Virol 1996, 70:3407-3415.

3. Dash B, Mclntosh A, Barrett W, Daniels R: Deletion of a single N-linked glycosylation site from the transmembrane envelope protein of human immunodeficiency virus type 1 stops cleavage and transport of gp160 preventing env-mediated fusion. J Gen Virol 1994, 75(Pt 6):1389-1397.

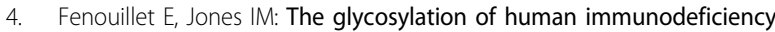
virus type 1 transmembrane glycoprotein (gp41) is important for the efficient intracellular transport of the envelope precursor gp160. $J$ Gen Virol 1995, 76(Pt 6):1509-1514.

5. Eisenberg $D$, Wesson $M$ : The most highly amphiphilic alpha-helices include two amino acid segments in human immunodeficiency virus glycoprotein 41. Biopolymers 1990, 29:171-177.

6. Kliger $Y$, Shai $Y$ : A leucine zipper-like sequence from the cytoplasmic tail of the HIV-1 envelope glycoprotein binds and perturbs lipid bilayers. Biochemistry 1997, 36:5157-5169.

7. Gawrisch K, Han KH, Yang JS, Bergelson LD, Ferretti JA: Interaction of peptide fragment 828-848 of the envelope glycoprotein of human immunodeficiency virus type I with lipid bilayers. Biochemistry 1993, 32:3112-3118.

8. Srinivas SK, Srinivas RV, Anantharamaiah GM, Segrest JP, Compans RW: Membrane interactions of synthetic peptides corresponding to amphipathic helical segments of the human immunodeficiency virus type-1 envelope glycoprotein. J Biol Chem 1992, 267:7121-7127.

9. Viard M, Ablan SD, Zhou M, Veenstra TD, Freed EO, Raviv Y, Blumenthal R: Photoinduced reactivity of the HIV-1 envelope glycoprotein with a membrane-embedded probe reveals insertion of portions of the HIV-1 Gp41 cytoplasmic tail into the viral membrane. Biochemistry 2008, 47:1977-1983.

10. Muesing MA, Smith DH, Cabradilla CD, Benton CV, Lasky LA, Capon DJ: Nucleic acid structure and expression of the human AIDS/ lymphadenopathy retrovirus. Nature 1985, 313:450-458.

11. Haffar OK, Dowbenko DJ, Berman PW: Topogenic analysis of the human immunodeficiency virus type 1 envelope glycoprotein, gp160, in microsomal membranes. J Cell Biol 1988, 107:1677-1687.

12. Yang C, Spies CP, Compans RW: The human and simian immunodeficiency virus envelope glycoprotein transmembrane subunits are palmitoylated. Proc Natl Acad Sci USA 1995, 92:9871-9875.

13. Rowell JF, Stanhope PE, Siliciano RF: Endocytosis of endogenously synthesized HIV-1 envelope protein. Mechanism and role in processing for association with class II MHC. J Immunol 1995, 155:473-488.

14. Kennedy RC, Henkel RD, Pauletti D, Allan JS, Lee TH, Essex M, Dreesman GR: Antiserum to a synthetic peptide recognizes the HTLV-III envelope glycoprotein. Science 1986, 231:1556-1559.

15. Vella C, Ferguson M, Dunn G, Meloen R, Langedijk H, Evans D, Minor PD: Characterization and primary structure of a human immunodeficiency virus type 1 (HIV-1) neutralization domain as presented by a poliovirus type 1/HIV-1 chimera. J Gen Virol 1993, 74(Pt 12):2603-2607.

16. Cleveland SM, McLain L, Cheung L, Jones TD, Hollier M, Dimmock NJ: A region of the C-terminal tail of the gp41 envelope glycoprotein of human immunodeficiency virus type 1 contains a neutralizing epitope: evidence for its exposure on the surface of the virion. J Gen Virol 2003, 84:591-602.

17. Lu L, Zhu Y, Huang J, Chen $X$, Yang $H$, Jiang S, Chen YH: Surface exposure of the HIV-1 env cytoplasmic tail LLP2 domain during the membrane fusion process: interaction with gp41 fusion core. J Biol Chem 2008, 283:16723-16731.

18. Yue L, Shang L, Hunter E: Truncation of the membrane-spanning domain of human immunodeficiency virus type 1 envelope glycoprotein defines elements required for fusion, incorporation, and infectivity. J Virol 2009, 83:11588-11598.

19. Shang $L$, Yue $L$, Hunter E: Role of the membrane-spanning domain of human immunodeficiency virus type 1 envelope glycoprotein in cell-cell fusion and virus infection. J Virol 2008, 82:5417-5428.

20. Salzwedel K, Johnston PB, Roberts SJ, Dubay JW, Hunter E: Expression and characterization of glycophospholipid-anchored human immunodeficiency virus type 1 envelope glycoproteins. J Virol 1993, 67:5279-5288.

21. Weiss $C D$, White JM: Characterization of stable Chinese hamster ovary cells expressing wild-type, secreted, and glycosylphosphatidylinositol- 
anchored human immunodeficiency virus type 1 envelope glycoprotein. J Virol 1993, 67:7060-7066.

22. Owens RJ, Burke C, Rose JK: Mutations in the membrane-spanning domain of the human immunodeficiency virus envelope glycoprotein that affect fusion activity. J Virol 1994, 68:570-574.

23. Miyauchi K, Komano J, Yokomaku Y, Sugiura W, Yamamoto N, Matsuda Z: Role of the specific amino acid sequence of the membrane-spanning domain of human immunodeficiency virus type 1 in membrane fusion. $J$ Virol 2005, 79:4720-4729.

24. Miyauchi K, Curran R, Matthews E, Komano J, Hoshino T, Engelman DM, Matsuda Z: Mutations of conserved glycine residues within the membrane-spanning domain of human immunodeficiency virus type 1 gp41 can inhibit membrane fusion and incorporation of Env onto virions. Jpn J Infect Dis 2006, 59:77-84.

25. Wang J, Kondo N, Long Y, Iwamoto A, Matsuda Z: Monitoring of HIV-1 envelope-mediated membrane fusion using modified split green fluorescent proteins. J Virol Methods 2009, 161:216-222.

26. Kondo N, Ebihara A, Ru H, Kuramitsu S, Iwamoto A, Rao Z, Matsuda Z: Thermus thermophilus-derived protein tags that aid in preparation of insoluble viral proteins. Anal Biochem 2009, 385:278-285.

27. Wilk T, Pfeiffer T, Bukovsky A, Moldenhauer G, Bosch V: Glycoprotein incorporation and HIV-1 infectivity despite exchange of the gp160 membrane-spanning domain. Virology 1996, 218:269-274.

28. Caras IW, Weddell GN, Williams SR: Analysis of the signal for attachment of a glycophospholipid membrane anchor. J Cell Biol 1989, 108:1387-1396

29. Cosson P, Lankford SP, Bonifacino JS, Klausner RD: Membrane protein association by potential intramembrane charge pairs. Nature 1991 , 351:414-416.

30. van Genderen H, Kenis H, Lux P, Ungeth L, Maassen C, Deckers N, Narula J, Hofstra L, Reutelingsperger $C$ : In vitro measurement of cell death with the annexin A5 affinity assay. Nat Protoc 2006, 1:363-367.

31. Drew D, Sjostrand D, Nilsson J, Urbig T, Chin CN, de Gier JW, von Heijne G: Rapid topology mapping of Escherichia coli inner-membrane proteins by prediction and PhoA/GFP fusion analysis. Proc Natl Acad Sci USA 2002, 99:2690-2695.

32. Duffy EB, Barquera B: Membrane topology mapping of the Nat-pumping $\mathrm{NADH}$ : quinone oxidoreductase from Vibrio cholerae by PhoA-green fluorescent protein fusion analysis. J Bacteriol 2006, 188:8343-8351.

33. Los GV, Encell LP, McDougall MG, Hartzell DD, Karassina N, Zimprich C, Wood MG, Learish R, Ohana RF, Urh M, et al: HaloTag: a novel protein labeling technology for cell imaging and protein analysis. ACS Chem Biol 2008, 3:373-382.

34. Kondo N, Miyauchi K, Meng F, Iwamoto A, Matsuda Z: Conformational changes of the HIV-1 envelope protein during membrane fusion are inhibited by the replacement of its membrane-spanning domain. J Biol Chem 2010, 285:14681-14688.

35. Cloyd MW, Lynn WS: Perturbation of host-cell membrane is a primary mechanism of HIV cytopathology. Virology 1991, 181:500-511.

36. Zhang $H$, Dornadula $G$, Alur P, Laughlin MA, Pomerantz RJ: Amphipathic domains in the $C$ terminus of the transmembrane protein (gp41) permeabilize HIV-1 virions: a molecular mechanism underlying natural endogenous reverse transcription. Proc Natl Acad Sci USA 1996, 93:12519-12524.

37. Gatti PJ, Choi B, Haislip AM, Fermin CD, Garry RF: Inhibition of HIV type 1 production by hygromycin B. AIDS Res Hum Retroviruses 1998, 14:885-892.

38. Voss TG, Fermin CD, Levy JA, Vigh S, Choi B, Garry RF: Alteration of intracellular potassium and sodium concentrations correlates with induction of cytopathic effects by human immunodeficiency virus. J Virol 1996, 70:5447-5454.

39. Miller MA, Cloyd MW, Liebmann J, Rinaldo CR, Islam KR, Wang SZ, Mietzner TA, Montelaro RC: Alterations in cell membrane permeability by the lentivirus lytic peptide (LLP-1) of HIV-1 transmembrane protein. Virology 1993, 196:89-100.

40. Chernomordik L, Chanturiya AN, Suss-Toby E, Nora E, Zimmerberg J: An amphipathic peptide from the C-terminal region of the human immunodeficiency virus envelope glycoprotein causes pore formation in membranes. J Virol 1994, 68:7115-7123.

41. Arroyo J, Boceta M, Gonzalez ME, Michel M, Carrasco L: Membrane permeabilization by different regions of the human immunodeficiency virus type 1 transmembrane glycoprotein gp41. J Virol 1995, 69:4095-4102.
42. Comardelle AM, Norris CH, Plymale DR, Gatti PJ, Choi B, Fermin CD, Haislip AM, Tencza SB, Mietzner TA, Montelaro RC, Garry RF: A synthetic peptide corresponding to the carboxy terminus of human immunodeficiency virus type 1 transmembrane glycoprotein induces alterations in the ionic permeability of Xenopus laevis oocytes. AIDS Res Hum Retroviruses 1997, 13:1525-1532.

43. Suarez T, Gallaher WR, Agirre A, Goni FM, Nieva JL: Membrane interfaceinteracting sequences within the ectodomain of the human immunodeficiency virus type 1 envelope glycoprotein: putative role during viral fusion. J Virol 2000, 74:8038-8047.

44. Suarez T, Nir S, Goni FM, Saez-Cirion A, Nieva JL: The pre-transmembrane region of the human immunodeficiency virus type-1 glycoprotein: a novel fusogenic sequence. FEBS Lett 2000, 477:145-149.

45. Brugger B, Glass B, Haberkant P, Leibrecht I, Wieland FT, Krausslich HG: The HIV lipidome: a raft with an unusual composition. Proc Natl Acad Sci USA 2006, 103:2641-2646.

46. Saez-Cirion A, Arrondo JL, Gomara MJ, Lorizate M, lloro I, Melikyan G, Nieva JL: Structural and functional roles of HIV-1 gp41 pretransmembrane sequence segmentation. Biophys J 2003, 85:3769-3780.

47. Saez-Cirion A, Nir S, Lorizate M, Agirre A, Cruz A, Perez-Gil J, Nieva JL: Sphingomyelin and cholesterol promote HIV-1 gp41 pretransmembrane sequence surface aggregation and membrane restructuring. J Biol Chem 2002, 277:21776-21785.

48. Apellaniz B, Nir S, Nieva JL: Distinct mechanisms of lipid bilayer perturbation induced by peptides derived from the membrane-proximal external region of HIV-1 gp41. Biochemistry 2009, 48:5320-5331.

49. Huarte N, Lorizate M, Kunert R, Nieva JL: Lipid modulation of membranebound epitope recognition and blocking by HIV-1 neutralizing antibodies. FEBS Lett 2008, 582:3798-3804.

50. Dimitrov AS, Rawat SS, Jiang S, Blumenthal R: Role of the fusion peptide and membrane-proximal domain in HIV-1 envelope glycoproteinmediated membrane fusion. Biochemistry 2003, 42:14150-14158.

\section{doi:10.1186/1742-4690-7-100}

Cite this article as: Liu et al:: Membrane topology analysis of HIV-1 envelope glycoprotein gp41. Retrovirology 2010 7:100.

\section{Submit your next manuscript to BioMed Central and take full advantage of:}

- Convenient online submission

- Thorough peer review

- No space constraints or color figure charges

- Immediate publication on acceptance

- Inclusion in PubMed, CAS, Scopus and Google Scholar

- Research which is freely available for redistribution

Submit your manuscript at www.biomedcentral.com/submit
C Biomed Central 